\title{
ADDITIVE PROPERTIES OF MULTIPLICATIVE SUBGROUPS OF FINITE INDEX IN FIELDS
}

\author{
PEDRO BERRIZBEITIA
}

(Communicated by Louis J. Ratliff, Jr.)

\begin{abstract}
Gallai's theorem, an $n$-dimensional generalization of Van der Waerden's theorem on arithmetic progression, is used to prove the following theorem:

Let $F$ be a field and $G \subseteq F^{*}$ a subgroup of finite index $n$. There is a positive integer $N$, which depends only on $n$, so that if Char $F=0$ or char $F \geq N$, then $G-G=F$.
\end{abstract}

\section{INTRODUCTION}

It is well known that if $n$ is a positive integer and $p$ is a prime number, then the equation $x^{n}+y^{n} \equiv t \bmod p$ has a solution for any $t$ as long as $p$ is sufficiently large. This problem can be solved by using Jacobi Sums to approximate the number of solutions of the equation. This is done in [3, Chapter 8]. The problem can be restated as follows: If $G$ is a subgroup of $F_{p}^{*}$ of index at most $n$, then, for large enough values of $p, G+G \supseteq F_{p}^{*}$, with equality if and only if $-1 \notin G$.

For the case $n=3$ Leep and Shapiro [5] were able to prove the following generalization. Let $G$ be a subgroup of index 3 in the multiplicative group $F^{*}$ of a field $F$. Then $G+G=F$, except in the cases $|F|=4,7,13$, or 16 . They also conjectured that the conclusion holds for infinite fields if the index is 5 .

In $\S 1$ of this paper we prove Theorem 1.1 which states: Let $F$ be a field and $G \supseteq F^{*}$ a subgroup of finite index $n$. There is a positive integer $N$, that depends only on $n$, so that if Char $F=0$ or $\operatorname{char} F \geq N$, then, $G-G=F$.

Fields for which the conclusion of Theorem 1.1 holds will be called uniform. It follows that if $F$ is a uniform field, then, $G+G=F$ if and only if $-1 \in G$. This implies, in particular, that the above conjecture is true for uniform fields. More generally, we show that if $m$ is a positive integer and $m \times G=\left\{g_{1}+\right.$ $\left.g_{2}+\cdots+g_{m} \mid g_{i} \in G\right\}$ then $m \times G=F$ if and only if $-1 \in m-1 \times G$ or, equivalently, if and only if $0 \in m \times G$ (see Corollary 1.2 below). In $\S 2$ we let $F$ be an arbitrary field and $G \subseteq F^{*}$ a subgroup of index $n$ in $F^{*}$. Let $P$ denote the additive closure of $G$ in $F$. We show that if $F$ is uniform, then

Received by the editors February 23, 1990 and, in revised form, May 7, 1990.

1980 Mathematics Subject Classification (1985 Revision). Primary 11P99; Secondary 12E99. 
$P=m \times G$ for some $m \leq[P: G]+1$. (See Corollary 2.2.) Finally, in $\S 3$ we let $\mathbf{Q}$ denote the field of the rational numbers. For every odd prime $p \geq 5$, we construct a subgroup of index $n=p-1$ in $\mathbf{Q}^{*}$, such that $n+1 \times G=\mathbf{Q}$ but $m \times G \neq \mathbf{Q}$ for all $m \leq n$.

\section{UNIFORM FIELDS}

We follow Leep and Shapiro's notation. Let $F$ be a field. Let $G \subseteq F^{*}$ be a subgroup of finite index $n$. For each positive integer $m$ we denote by $m \times G$ the set of sums of $m$ elements of $G$; that is, $m \times G=\left\{g_{1}+g_{2}+\cdots+g_{m} \mid g_{i} \in\right.$ $G\}$. The additive closure of $G$ will be denoted by $\sum G$ or simply $P$. So $P=\bigcup_{i=1}^{\infty} i \times G . P_{m}$ will denote the partial union $P_{m}=\bigcup_{i=1}^{m} i \times G$. Finally let $G-G=\left\{g_{1}-g_{2} \mid g_{i} \in G\right\}$. We now state the main theorem of this paper.

Theorem 1.1. Let $F$ be a field and $G \subseteq F^{*}$ a subgroup of finite index $n$. There is a positive integer $N$, which depends only on $n$, so that if Char $F=0$ or char $F \geq N$, then, $G-G=F$.

For simplicity, fields for which the conclusion of Theorem 1.1 holds will be called uniform with respect to subtraction or simply uniform fields.

Proof of Theorem 1.1. We make some preliminary remarks. Note first that $G-G=F$ if and only if $c G-c G=F$ for any $c \in F^{*}$. Also note that $c G-c G$ consists of a union of cosets of $F^{*} \bmod G$ and of 0 . Hence, to prove that $G-G=F$, it is enough to show that $c G-c G$ contains a complete set of representatives of $F^{*} \bmod G$ for some $c \in F$.

Let $\mathbf{N}$ be the set of nonnegative integers. For $m \in \mathbf{N}, m$ positive, let $\mathbf{N}^{m}=\left\{\left(x_{1}, x_{2}, \ldots, x_{m}\right) \mid x_{i} \in \mathbf{N}\right\}$. We will use the following result due to Gallai:

Theorem G. Let $V \subseteq \mathbf{N}^{m}$ be finite. For any finite coloring of $\mathbf{N}^{m}$ there is $a \in \mathbf{N}^{m}$ and nonzero $d \in \mathbf{N}$, so that all the points of $a+d V$ have the same color.

Gallai's theorem is a result of Ramsey theory. It is derived from the HalesJewett theorem in [2]. To show the existence of $N(n)$ in Theorem 1.1, we will use the following apparently stronger but equivalent form of Gallai's theorem. For $s \in N$ let $[s]=\{0,1,2, \ldots, s\}$.

Theorem $\mathbf{G}^{\prime}$. Let $V \subseteq \mathbf{N}^{m}$ be finite. Let $m \in \mathbf{N}$ be positive. There is a natural number $N^{\prime}=N^{\prime}(V, m, r)$, such that for any $N \geq N^{\prime}$ and any $r$-coloring of $[N]^{m}$, there is $a \in\left[N^{\prime}\right]^{m}$ and a nonzero $d \in \mathbf{N}, d \leq N^{\prime}$, such that $a+d V$ is monochromatic.

That Theorem $G^{\prime}$ is equivalent to Theorem $G$ is a consequence of König's infinity lemma. This modification was first introduced by O. Schreier for the case $m=1$. See [6].

In our situation we assume $m=n$, the index of $G$ in $F^{*} . V \in \mathbf{N}^{n}$ will be the set $V=\left\{0, e_{1}, e_{2}, \ldots, e_{n}\right\}$, where the $e_{i}$ are the vectors of the canon- 
ical basis for $\mathbf{R}^{n}$. To each one of the cosets of $F^{*} \bmod G$ we associate a different color and to $0 \in F$ we associate another color. We will induce a $(n+1)$-coloring on the set $\mathbf{N}^{n}$ as follows: Let $g_{1}, g_{2}, \ldots, g_{n}$ be a set of representatives of $F^{*} \bmod G$. Paint each $\left(x_{1}, x_{2}, \ldots, x_{n}\right) \in \mathbf{N}^{n}$ with the color associated to the class of $x_{1} g_{1}+x_{2} g_{2}+\cdots+x_{n} g_{n}$. By Gallai's theorem, there is $a=\left(a_{1}, a_{2}, \ldots, a_{n}\right) \in \mathbf{N}^{n}$ and a nonzero $d \in \mathbf{N}$ such that $a+d V$ is monochromatic. It follows that if $c=a_{1} g_{1}+a_{2} g_{2}+\cdots+a_{n} g_{n}$, then $c$ and $c+d g_{i}$ have the same color associated. Hence $d g_{i} \in c G-c G$ for all $i=1,2, \ldots, n$. If $d \neq 0$ in $F$ the $d g_{i}$ run through all classes of $F^{*} \bmod G$. In this case, by the preliminary remarks we get $G-G=F$. Clearly $d$ is nonzero in $F$ when $F$ has characteristic zero. Also, since $d \leq N^{\prime}=N^{\prime}(V, n, n+1)$ given by Theorem $\mathrm{G}^{\prime}$ the same conclusion holds for fields of characteristic larger than $N^{\prime}$. Since $V$ depends only on $n$ we let $N(n)=N^{\prime}$.

Remark. Following the same techniques used in [1] to derive a proof of Van der Waerden's theorem from the Hales-Jewett theorem, one can derive from HalesJewett a particular case of Gallai's theorem that is enough to prove Theorem 1.1. If this is done, one can show that $N(n)$ can be chosen to be no larger than $2^{M}$ where $M=N\left(n+1,2^{n}\right)$ is the number given in the Hales-Jewett theorem. The numbers $N(r, t)$ are defined recursively and, therefore are computable, however, they are already extremely large for small values of $t$ and $r$.

Corollary 1.2. (a) Let $F$ be a uniform field. If $m>1$, then $m \times G=F$ if and only if $-1 \in m-1 \times G$. In particular $G+G=\mathbf{F}$ if and only if $-1 \in G$.

(b) If $G$ has odd index in $F^{*}$ for some uniform field $F$, then $G+G=F$.

Proof. If $m \times G=F$ then $g_{1}+g_{2}+\cdots+g_{m}=0$ for some $g_{i} \in G$. Then $-1=$ $g_{2} g_{1}^{-1}+g_{3} g_{1}^{-1}+\cdots+g_{m} g_{1}^{-1} \in m-1 \times G$. For the converse, if $-1 \in m-1 \times G$ then $-G \subseteq m-1 \times G$. Using Theorem 1.1 we have: $F=G-G=G+(-G) \subseteq$ $G+m-1 \times G=m \times G$. This proves (a). For (b) note that if $\left[F^{*}: G\right]=2 k+1$ then $(-1)^{2 k+1}=-1 \in G$. The result follows from (a). (The case $n=5$ was conjectured in [5] for arbitrary finite fields.)

Remark. Part (a) of Corollary 1.2 could have been stated in the following equivalent form: If $F$ is a uniform field, then $m \times G=F$ if and only if $0 \in m \times G$.

\section{INEQUALITIES CONCERNING SETS OF SUMS OF ELEMENTS OF $G$}

Let $F$ be an arbitrary field and $G \subseteq F^{*}$ be a multiplicative subgroup of finite index. Lemma 1 of [5] shows that if $-1 \in P$ then $P=F$. In any case, we have the following:

Proposition 2.1. If $G$ has index $n$ in $F^{*}$ we have:

(a) For $i \geq 1, P_{i} \subseteq P_{i+1}$ with equality if and only if $P_{i}=P$.

(b) If $-1 \in P$ then $P_{n+1}=F$. If $-1 \notin P$ then $P_{(n / 2)+1}=P$.

Proof. $P_{i} \subseteq P_{i+1}$ by definition of $P_{i}$. If $P_{i}=P$ then $P_{i+1}=P_{i}+G=P+G=$ $P=P_{i}$, so $P_{i+1} \subseteq P_{i}$. Conversely, if $P_{i}=P_{i+1}$ then $P_{i}=P_{r}$ for all $r>i$. 
But $P=\bigcup_{r=1}^{\infty} P_{r}=P_{i}$. For (b) just note that every $P_{m}$ is a union of cosets of $F^{*}$ and possibly of $\{0\}$. For simplicity $\{0\}$ is considered to be a coset. Suppose that $-1 \in P$ and that $P_{n} \neq F$. Then, by part (a), for all $i \leq n$, $P_{i+1}$ contains at least one class more than $P_{i}$. It follows that $P_{n+1}$ contains all classes including $\{0\}$. So $P_{n+1}=F$. If $-1 \notin P,\left[F^{*}: P\right] \geq 2$ then $[P: G] \leq n / 2$ and the argument is the same as before.

Corollary 2.2. If $F$ is a uniform field we have:

(a) $i \times G \subseteq i+1 \times G$ with equality if and only if $i \times G=P$.

(b) If $-1 \in P$ then $n+1 \times G=F$. If $-1 \notin P$ then $n / 2+1 \times G=P$.

Proof. Using Proposition 3.1 we see that it is enough to show that $i \times G=P_{i}$ for any $i$. For this we note that it suffices to show that $G \subseteq G+G$. But, since $F$ is uniform, then $G-G=F$. So $G-G \supseteq G$. This means that there are elements $g_{1}, g_{2}, g_{3}$ in $G$ such that $g_{1}-g_{2}=g_{3}$. So $g_{1}=g_{2}+g_{3}$, which implies $G+G \subseteq G$.

Remark. In general it is not true that $n+1 \times G=P$. Let $F=F_{7}$, the finite field with 7 elements and let $G=\{1,-1\}$. Then $\left[F^{*}: G\right]=3$ and $4 \times G \neq F$.

\section{BOUNDS IN $\S 2$ CANNOT BE IMPROVED}

We end this paper with examples of subgroups of finite index $n$ in $\mathbf{Q}^{*}$ for which $n+1$ is the minimum $r$ such that $\mathbf{Q}=r \times G$. We note that subgroups of finite index in $\mathbf{Q}^{*}$ are kernels of multiplicative maps of $\mathbf{Q}^{*}$ into finite groups. These maps are determined by the image of the prime numbers and by the image of -1 .

Let $q$ be an odd prime $q \geq 5$. We will map $\mathbf{Q}^{*}$ into $F_{q}^{*}$ as follows: if $p \neq q$ is a prime then let $p$ be mapped to its class $\bmod q$. Let the prime $q$ be mapped into 1 and let -1 be mapped to its class $\bmod q$. This way every rational number which is a unit in the field of the $q$-adic number is mapped into its class $\bmod q$. Hence, if $G$ is the kernel of this map, we have, $n \in G$ if and only if $n=q^{r} u_{1}$, where $r$ is an integer and $u_{1}$ is a unit, $u_{1} \equiv 1 \bmod q$. It is then easy to verify that $q-1 \times G=\mathbf{Q}^{*}$. But $q-1$ is the index of $G$ in $\mathbf{Q}^{*}$.

\section{ACKNOWLEDGMENT}

I am grateful to Professor D. B. Shapiro for telling me about Van der Waerden's $n$-dimensional generalization. My original version of Theorem 1.1 used the classical one-dimensional Van der Waerden's theorem and involved only the case $F=\mathbf{Q}$, the field of rational numbers.

\section{REFERENCES}

1. R. L. Graham, Rudiments of Ramsey theory, Regional Conf. Ser. in Math., vol. 45, Amer. Math. Soc., Providence, RI, 1979.

2. R. L. Graham, B. L. Rothschild, and J. H. Spencer, Ramsey theory, Wiley-Interscience, 1980. 
3. K. Ireland and M. Rosen, $A$ classical introduction to modern number theory, Springer-Verlag, Berlin, 1982.

4. A. Y. Khinchin, Three pearls of number theory, Rochester, NY, 1952, pp. 11-17.

5. D. B. Leep and D. B. Shapiro, Multiplicative subgroups of index three in a field, Proc. Amer. Math. Soc. 105 (1989), 802-807.

6. B. L. Van der Waerden, How the proof of Baudet's conjecture was found, Studies in Pure Mathematics (L. Mirsky, ed.), Academic Press, London, 1971, pp. 251-260.

Departamento de Matemáticas Puras y Aplicadas, Universidad Simón Bolivar, Apartado 89000, Caracas 1080-A, Venezuela 